\title{
Efficacy of Some Anticoccidial Drugs on Experimentally Induced Cecal Coccidiosis $(E$. tsunodai) in Japanese Quails
}

\author{
M.A. El-Morsy, K.I. Abou El-Azm and S.S. Awad \\ Department of Poultry and Rabbit Diseases, Faculty of \\ Veterinary Medicine, Mansoura University, Egypt.
}

OCYSTS of different Eimeria species were isolated from 27
isolated from 27 farms of Japanese quail which collected from
which collected from Al-Dakahlia and Kafr El-Sheikh provinces,
Egypt. Eimeria isolates were identified as E. bateri, E. uzura and E.
tsunodai in addition to 12 isolates of an unidentified Eimeria species.
Efficacy of coccidial prophylactic feed additives (salinomycin and
diclazuril) and coccidial water medicaments (amprolium, ethopabate
and toltrazuril) were studied in Japanese quails experimentally
infected at fourteenth days old with $4.1 \times 10^{4}$ E. tsunodai sporulated
oocysts. All groups which inoculated with E. tsunodai and received
any one of the tested drugs had better clinical signs, mortality rate,
lesion scores, oocyst output, sporulation percent, weight gain, feed
consumption and FCR than the infected non treated group. Although
all tested anticoccidials significantly improved the adverse effects of
coccidia, yet there was no absolute superiority of any tested drug than
the others. Generally we can say that water medicaments gave much
better results in E. tsunodai infected quails than the feed additive
anticoccidials.

Keywods: Coccidia, Eimeria tsunodai, Quil, Salinomycin, Treatment.

Breeding of Japanese quail (Coturnix japonica) excelled in aviculture due to increased consumption of exotic meats and eggs and has been considered as an alternative to chicken production (Berto et al., 2013).

Japanese quails are susceptible to various diseases such as coccidiosis which regarded as a serious parasitic disease problem limiting quuail industry (Seok et al., 2003).

Coccidiosis affects economical parameters (weight gain, body weight, FCR) in addition it induce immune suppression and the increase mortality of other diseases.

The aim of this work is to investigate different types of coccidia infected quail farms in Al-Dakahlia and Kafr El-Seikh provinces and to evaluate the efficacy of some anticoccidials such as salinomycine and diclazuril as preventive medicaments in ration and treatment drugs as toltrazuuril and amprolium plus ethopabate in water on cecal coccidiosis (E. tsunodai) in Japanese quails. 
Materials

\section{Material and Methods}

Experimental birds

Two hundred and forty 1- day old Japanese quail chicks were purchased from local hatchery for experimental study.

Ration: Starter and finisher rations free from antibiotics and anticoccidial drugs were used in the experimental study.

Anticoccidial drugs :

- Coccidiostac ${ }^{\circledR}$ (Salinomycin sodium 6\%): UCCMA company, batch NO. 49/B/2/2015.

- Diclazuril 0.5\%® (Diclazuril) : KEPRO B.V. company, batch NO. 20141210.

- AMERIL ${ }^{\circledR}$ (Amprolium HCL plus ethopabate) : Waki Pharma company, batch NO. 0060115.

- DADCOX 2.5\%® (Toltrazuril) : Intercova company, batch NO.151204.

\section{Methods}

Isolation and identification of coccidia from quail farms

One hundred and eight samples were collected from Japanese quail farms from different localities in Al-Dakahlia and Kafr El-Sheikh provinces. Specimens from fecal contents and mucosa were taken from duodenum, jejunum, ileum and cecum. Samples were examined in direct smear (Urquhart, 2003) and by floatation technique under light microscope (10x) (Duszynski and Wilber, 1997). Morphometric identification was done by using calibrated ocular micrometer according to Henddrix and Robinson (2006).

Oocysts were prepared and concentrated according to Soulsby (1968). Oocysts were sporulated as described by Adefolabi and Chiejina (1987) and Harper and Penzorn (1999) and preserved as mentioned by Norton and Joyner (1968).

Propagation of coccidial isolates

One day-old Japanese quail chicks were reared in isolated sterilized wire floored rearing cages. Quails were fed autoclaved anticoccidials free ration and drink autoclaved water. They were inoculated intra crop by $4.1 \times 10^{4}$ oocysts of E. tsunodai oocysts (field isolate).

All quails were sacrificed on day 6 post infection (P.I). E. tsunodai oocysts were collected from cecal contents. Collected oocysts were sporulated and preserved as described by Norton and Joyner (1968) to be used for experimental infection.

Oocysts counting

Oocysts were counted by using McMaster-chamber method according to Conway and Mckenzie (1991).

Egypt. J. Vet. Sci. Vol. 47, No.2 (2016) 
Lesion scores

Severity of lesions was evaluated according to Johnson and Reid (1970). Range of lesion scores was between 0 and +4 .

\section{Experimental design}

Two hundred and forty one-day old Japanese quails were used to evaluate the efficacy of four anticoccidials on E. tsunodai. Quails were randomly distributed in wire-floored rearing cages into eight groups that were sub divided at tenth days old into three replicates per group. Groups were treated as follow : Group (1): Negative control group (non-infected non-treated).

Group (2): E. tsunodai positive control group. Quails were experimentally infected with $4.1 \times 10^{4}$ sporulated oocysts of E. tsunodai at fourteenth days old without medication.

Group (3): E. tsunodai infected salinomycin medicated group. Quails were experimentally infected with $4.1 \times 10^{4}$ sporulated oocysts of E. tsunodai at fourteenth days old and fed ration containing salinomycin (Coccidiostac) (1 kg/ton) (60 ppm) after thorough mixing 48 hours before inoculation till 21 days post infection.

Group (4): Salinomycin control (non-infected salinomycin treated) group. Quails were fed on ration containing salinomycin (Coccidiostac) (1kg/ton) (60 ppm) at twelfth day old till the end of experiment.

Group (5): E. tsunodai infected diclazuril medicated group. Quails were experimentally infected with $4.1 \times 10^{4}$ sporulated oocysts of E. tsunodai on fourteenth day of age and fed ration containing diclazuril (Diclazuril 0.5\%) (200 $\mathrm{gm} /$ ton) after thorough mixing 48 hours before inoculation till 21 days post infection.

Group (6): Diclazuril control (non-infected diclazuril treated) group. Quails fed ration containing diclazuril $(200 \mathrm{~g} / \mathrm{ton})$ on day 12 of age till the end of experiment.

Group (7): E. tsunodai infected amprolium in addition to ethobapate treated group. Quails were experimentally infected with $4.1 \times 10^{4}$ sporulated oocysts of E. tsunodai on fourteenth day old and treated with amprolium and ethopabate (AMERIL) 48 hours post infection ( $1 \mathrm{ml} /$ liter for 5 days).

Group (8): E. tsunodai infected toltrazuril treated group. Quails were experimentally infected with $4.1 \times 10^{4}$ sporulated oocysts of $E$. tsunodai on day 14 of age and treated with toltrazuril (DADCOX 2.5\%) 48 hours post infection (25 ppm) (1 $\mathrm{ml} /$ litter) for two consecutive days.

Clinical signs and mortalities of each group were recorded throughout the period of experiment. Daily collection of fresh droppings from each experimental group was done twice daily at fixed times. Emeria oocysts counting started from the $4^{\text {th }}$ day post infection till the end of 21 days post infection.

On seventh day post infection, two quails from each replicate were randomly selected and sacrificed for examination of cecal lesions, lesion score and direct 
wet smears on cecal mucosa to detect the developmental stages of Eimeria. Carcass was opened and examined for the previous mentioned parameters.

Birds of each replicate were weighted individually on day 14 of age and thereafter on a weekly basis. Average feed intake, weight gain and FCR were calculated on a weekly basis. Statistical analysis

Data were statistically analyzed by analysis of variance (ANOVA), using least significant difference (LSD) as described by Snedecor and Cochran (1981).

\section{Results}

Oocysts of different Eimeria species were isolated from 27 Japanese quail farms present in Al-Dakahlia and Kafr El-Sheikh provinces. Eimeria isolates were identified as intestinal coccidiosis (E. bateri, Fig. 1 and E. uzura, Fig. 2), cecal coccidiosis (E. tsunodai, Fig. 3) and an unidentified Eimeria species, Fig. 4.
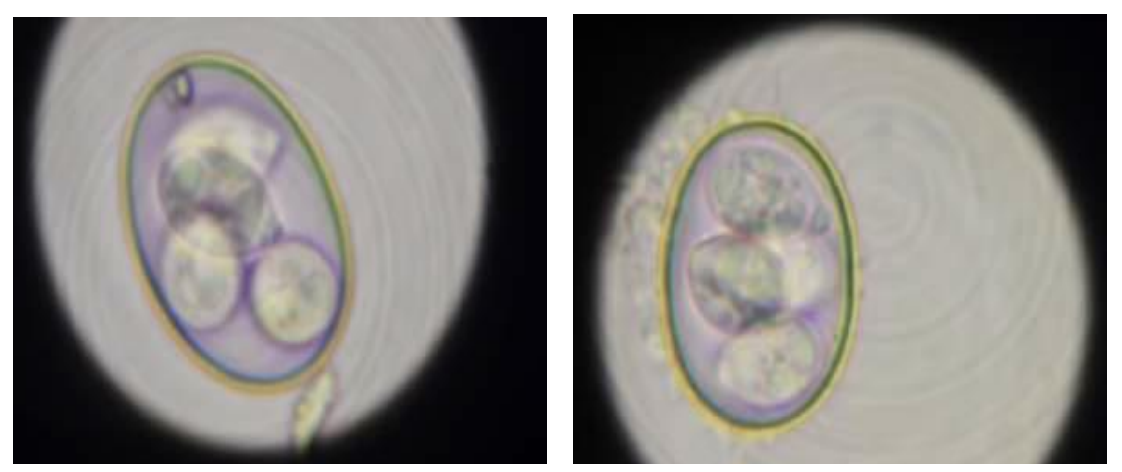

Fig. 1. shows E. bateri sporulated oocyst in saturated sugar solution (40x).
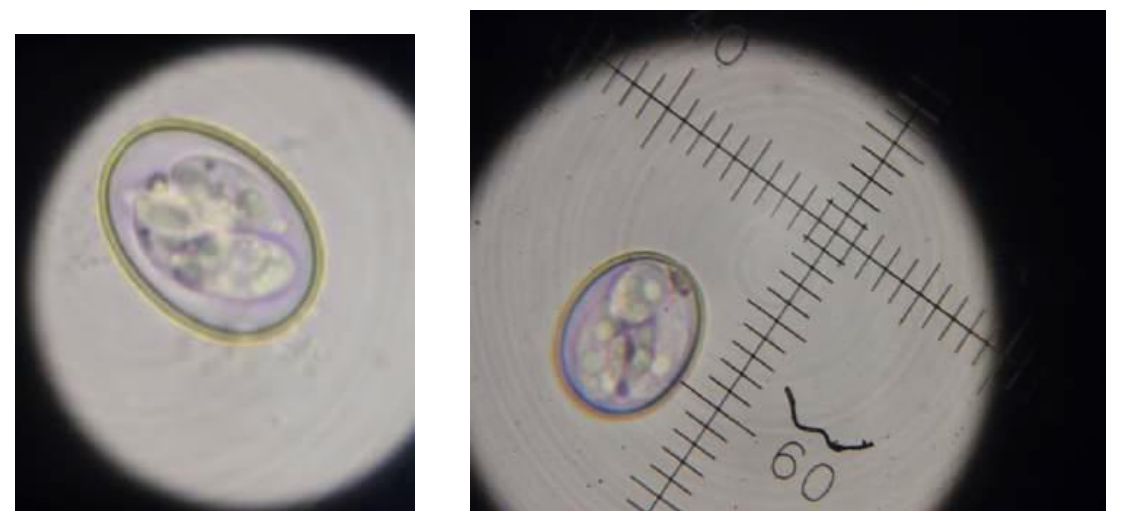

Fig. 2. Shows E. uzura sporulated oocyst in saturated sugar solution (40x).

Egypt. J. Vet. Sci. Vol. 47, No.2 (2016) 

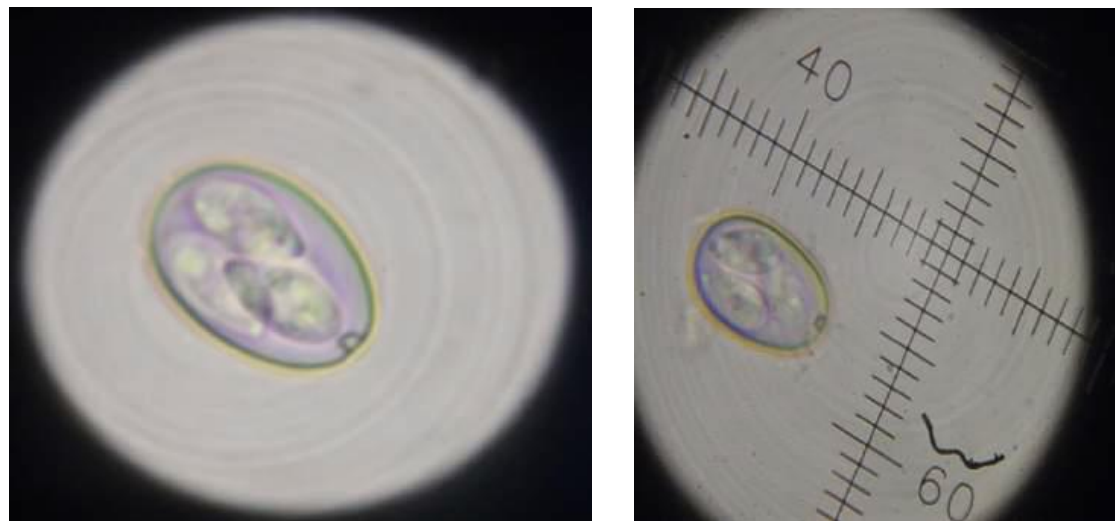

Fig. 3. Shows E. tsunodai sporulated oocyst in saturated sugar solution (40x).
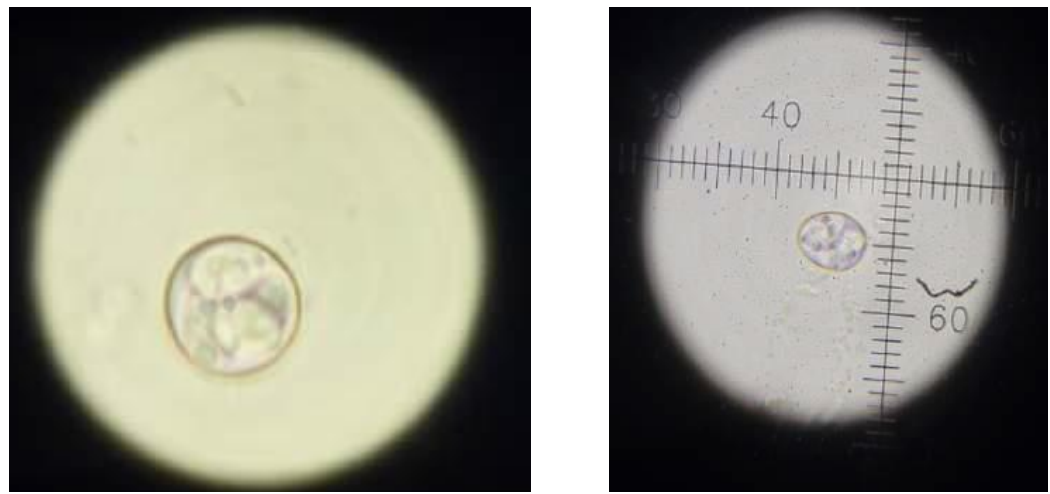

Fig. 4. Shows very small unidentified Eimeria sporulated oocyst in saturated sugar solution $(40 x)$.

Clinical signs appeared at the $4^{\text {th }}$ day post experimental infection. During the whole experimental period, non-infected non-treated group (negative control), non-infected salinomycin treated group (salinomycin control) and non-infected diclazuril treated group (diclazuril control) were completely healthy without mortality, normal dropping, normal appetite and good (body weight, weight gain and FCR values). Good intestinal and fecal examination revealed that no accidental coccidial infection was occurred in such group.

Infected non-treated group (positive control) was severely affected and showed the worst results with regard to clinical signs, mortality rate (Table 1), lesion score (Table 2), oocyst output (Table 3), weight gains (Table 4), FCR values (Table 5), body weights (Table 6) and sporulation percent (Table 7) in comparison with all infected and treated birds.

Egypt. J. Vet. Sci. Vol. 47, No.2 (2016) 
Symptoms were obvious in positive control group such as ruffled feather, depression, huddle together, decrease appetite, emaciated breast muscle, knife edged keel bone and bloody diarrhea. Severely enlarged two cecae with thickened mucosa, bloody cecal core and blooning were the most prominent lesions post E. tsunodai infection.

Diclazuril treated group showed better results than that of salinomycin in regard to all tested parameters except that both groups were showed the same lesion score with lower oocyst output in salinomycin treated group.

TABLE 1. Daily mortalities post $E$. tsunodai infection.

\begin{tabular}{|c|c|c|c|c|c|c|c|c|}
\hline \multirow{2}{*}{ 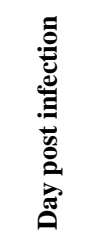 } & \multicolumn{8}{|c|}{ Daily mortalities } \\
\hline & 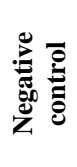 & $\underbrace{0}_{0}$ & 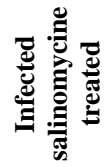 & 胥 & 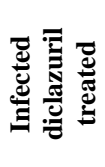 & 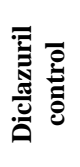 & 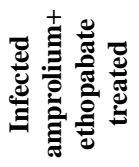 & 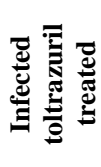 \\
\hline $5^{\text {th }}$ & 0 & 3 & 1 & 0 & 1 & 0 & 0 & 2 \\
\hline $6^{\text {th }}$ & 0 & 1 & 2 & 0 & 2 & 0 & 2 & 1 \\
\hline $7^{\text {th }}$ & 0 & 2 & 1 & 0 & 0 & 0 & 1 & 0 \\
\hline $8^{\text {th }}$ & 0 & 1 & 1 & 0 & 0 & 0 & 0 & 0 \\
\hline Total & 0 & 7 & 5 & 0 & 3 & 0 & 3 & 3 \\
\hline $\begin{array}{l}\text { Percent } \\
(\%)\end{array}$ & 0 & 23.3 & 16.7 & 0 & 10 & 0 & 10 & 10 \\
\hline
\end{tabular}

TABLE 2. Lesion scores $(0: 3)$ post $E$. tsunodai infection.

\begin{tabular}{|c|c|c|c|c|c|c|c|c|}
\hline \multirow[b]{2}{*}{ 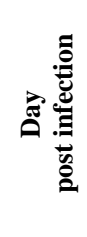 } & \multicolumn{8}{|c|}{ Lesion scores } \\
\hline & 㺼 & 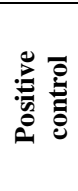 & 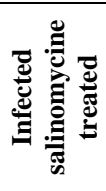 & 总 & 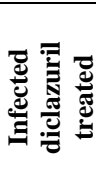 & 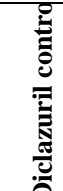 & 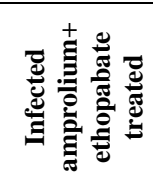 & 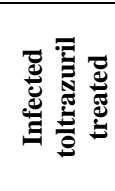 \\
\hline $5^{\text {th }}$ & - & +3 & +1 & - & +1 & - & - & +1 \\
\hline $6^{\text {th }}$ & - & +3 & +2 & - & +2 & - & +1 & +1 \\
\hline $7^{\text {th }}$ & 0 & +3 & +2 & 0 & +2 & 0 & +1 & +2 \\
\hline $8^{\text {th }}$ & - & +2 & +2 & - & - & - & - & - \\
\hline
\end{tabular}

Two birds from each replicate sacrificed on $7^{\text {th }}$ day post infection for detection of lesion score.

(-): No mortality.

Score 0: No gross lesion, normal contents.

Score +1 : Few scattered petechiae seen on the surface of the mucosa with little change in the color of the cecal wall or contents.

Score +2 : Presence of more blood and lesions, slight thickening of the cecal wall.

Score +3 : Sever bleeding, clotted blood and bloody dropping may occur.

Score +4 : Sever hemorrhage, expanded purplish ceca containing large clumps of clotted blood and whitish masses. 
TABLE 3. Daily oocyst output post $E$. tsunodai infection.

\begin{tabular}{|c|c|c|c|c|c|}
\hline \multirow[b]{2}{*}{$\begin{array}{c}\text { Day } \\
\text { post } \\
\text { infection }\end{array}$} & \multicolumn{5}{|c|}{ Daily oocyst output } \\
\hline & $\begin{array}{l}\text { Positive } \\
\text { control }\end{array}$ & $\begin{array}{c}\text { Infected } \\
\text { salinomycine } \\
\text { treated }\end{array}$ & $\begin{array}{c}\text { Infected } \\
\text { diclazuril } \\
\text { treated }\end{array}$ & $\begin{array}{c}\text { Infected } \\
\text { amprolium+ } \\
\text { ethopabate } \\
\text { treated }\end{array}$ & $\begin{array}{l}\text { Infected } \\
\text { toltrazuril } \\
\text { treated }\end{array}$ \\
\hline $4^{\text {th }}$ & 0 & 0 & 0 & 0 & 0 \\
\hline $5^{\text {th }}$ & $1047.7 \pm 28.434^{\mathrm{a}}$ & $553.2 \pm 74.5^{b}$ & $653.2 \pm 33.8^{\mathrm{b}}$ & $237.6 \pm 48.5^{\mathrm{c}}$ & $344 \pm 29.5^{c}$ \\
\hline $6^{\text {th }}$ & $1226 \pm 41.6^{\mathrm{a}}$ & $612.8 \pm 16.2^{\mathrm{b}}$ & $748 \pm 74.5^{\mathrm{b}}$ & $279.8 \pm 43.4^{\mathrm{c}}$ & $390.3 \pm 89.9^{c}$ \\
\hline $7^{\text {th }}$ & $678.8 \pm 23.3^{\mathrm{a}}$ & $410.8 \pm 51.3^{b}$ & $434.1 \pm 45.2^{\mathrm{b}}$ & $178 \pm 28.9^{c}$ & $246.7 \pm 47.8^{\mathrm{c}}$ \\
\hline $8^{\text {th }}$ & $476.3 \pm 25.4^{\mathrm{a}}$ & $202.7 \pm 2.8^{\mathrm{b}}$ & $204.7 \pm 3.03^{b}$ & $110.1 \pm 6.3^{\mathrm{c}}$ & $170 \pm 16.9^{b}$ \\
\hline $9^{\text {th }}$ & $245.6 \pm 25.9^{\mathrm{a}}$ & $95.8 \pm 4.3^{\mathrm{b}}$ & $96.1 \pm 7.2^{\mathrm{b}}$ & $62.8 \pm 3.6^{\mathrm{b}}$ & $80.3 \pm 3.6^{b}$ \\
\hline $10^{\text {th }}$ & $115.4 \pm 26.6^{a}$ & $71 \pm 5.9^{\mathrm{b}}$ & $74 \pm 5.4^{\mathrm{b}}$ & $47.5 \pm 3.6^{c}$ & $63.2 \pm 4.5^{\mathrm{b}}$ \\
\hline $11^{\text {th }}$ & $93.7 \pm 3.4^{\mathrm{a}}$ & $43 \pm 3.9^{b}$ & $44.8 \pm 4.2^{\mathrm{b}}$ & $25.7 \pm 2.8^{\mathrm{c}}$ & $37.2 \pm 2.3^{\mathrm{b}}$ \\
\hline $12^{\text {th }}$ & $71.9 \pm 3.5^{\mathrm{a}}$ & $29.5 \pm 2.8^{b}$ & $30.7 \pm 2.9^{b}$ & $19.8 \pm 2.5^{\mathrm{c}}$ & $27.4 \pm 2.1^{\mathrm{bc}}$ \\
\hline $13^{\text {th }}$ & $50 \pm 0.6^{\mathrm{a}}$ & $21.7 \pm 1.7^{\mathrm{bc}}$ & $22.8 \pm 2.2^{\mathrm{b}}$ & $13.6 \pm 2.3^{\mathrm{d}}$ & $16 \pm 2.1^{\mathrm{cd}}$ \\
\hline $14^{\text {th }}$ & $26.5 \pm 1.7^{\mathrm{a}}$ & $12.8 \pm 1.3^{b}$ & $14.3 \pm 1.3^{b}$ & $7.9 \pm 1.2^{\mathrm{c}}$ & $8.6 \pm 0.9^{c}$ \\
\hline $15^{\text {th }}$ & $12.6 \pm 0.8^{a}$ & $5.2 \pm 6.1^{\mathrm{bc}}$ & $5.8 \pm 0.5^{b}$ & $1.7 \pm 0.5^{\mathrm{d}}$ & $3.6 \pm 0.5^{\mathrm{cd}}$ \\
\hline $16^{\text {th }}$ & $3.8 \pm 0.9^{\mathrm{a}}$ & $1.3 \pm 0.4^{\mathrm{b}}$ & $1.9 \pm 0.5^{b}$ & $0.9 \pm 0.1^{\mathrm{b}}$ & $1.1 \pm 0.4^{\mathrm{b}}$ \\
\hline $17^{\text {th }}$ & $1.2 \pm 0.1^{\mathrm{a}}$ & $0.6 \pm 0.1^{\mathrm{bc}}$ & $0.7 \pm 0.1^{\mathrm{b}}$ & $0.2 \pm 0.03^{\mathrm{d}}$ & $0.4 \pm 0.1^{\mathrm{cd}}$ \\
\hline $18^{\text {th }}$ & $0.8 \pm 0.0^{\mathrm{a}}$ & $0.1 \pm 0.03^{\mathrm{b}}$ & $0.2 \pm 0.03^{\mathrm{b}}$ & $0.1 \pm 0.03^{\mathrm{b}}$ & $0.1 \pm 0.03^{\mathrm{b}}$ \\
\hline $19^{\text {th }}$ & $0.5 \pm 0.6^{\mathrm{a}}$ & $0.3 \pm 0.1^{\mathrm{b}}$ & $0.2 \pm 0.1^{\mathrm{bc}}$ & $0.1 \pm 0.1^{\mathrm{c}}$ & $0.1 \pm 0.1^{\mathrm{c}}$ \\
\hline $20^{\text {th }}$ & $0.4 \pm 0.03^{\mathrm{a}}$ & $0.1 \pm 0.1^{\mathrm{b}}$ & $0.2 \pm 0.03^{\mathrm{b}}$ & $0.1 \pm 0.00^{\mathrm{b}}$ & $0.1 \pm 0.0^{\mathrm{b}}$ \\
\hline $21^{\mathrm{st}}$ & $0.3 \pm 0.03^{\mathrm{a}}$ & $0.2 \pm 0.1^{\mathrm{ab}}$ & $0.2 \pm 0.1^{\mathrm{ab}}$ & $0.1 \pm 0.1^{\mathrm{b}}$ & $0.2 \pm 0.03^{\mathrm{a} b}$ \\
\hline
\end{tabular}

Means: Mean of 3 replicates (10 birds / replicate)

(Mean \pm std. error) x 1000

a $\mathrm{b}$ c d e $=$ Means in the same column with different superscripts are significantly different at $\mathrm{P} \leq 0.05$.

TABLE 4. Weekly weight gain (gm) post E. tsunodai infection.

\begin{tabular}{|l|c|c|c|}
\hline \multirow{2}{*}{ Treatments } & \multicolumn{3}{c|}{ Weekly weight gain (gm) } \\
\cline { 2 - 4 } & $\mathbf{1}^{\text {st }}$ Week & $\mathbf{2}^{\text {nd }}$ Week & $\mathbf{3}^{\text {rd }} \mathbf{W e e k}^{*}$ \\
\hline Negative control & $32.07 \pm 2.61^{\mathrm{b}}$ & $49.50 \pm 2.08^{\mathrm{ab}}$ & $69.67 \pm 0.20^{\mathrm{a}}$ \\
\hline Positive control & $14.17 \pm 2.09^{\mathrm{e}}$ & $32.80 \pm 2.53^{\mathrm{e}}$ & $49.77 \pm 0.55^{\mathrm{c}}$ \\
\hline Infected salinomycine treated & $18.46 \pm 1.99^{\mathrm{de}}$ & $34.67 \pm 2.98^{\mathrm{de}}$ & $52.40 \pm 2.54^{\mathrm{c}}$ \\
\hline Salinomycine control & $32.69 \pm 2.21^{\mathrm{b}}$ & $51.20 \pm 0.12^{\mathrm{ab}}$ & $72.30 \pm 0.20^{\mathrm{a}}$ \\
\hline Infected diclazuril treated & $22.97 \pm 0.60^{\mathrm{cd}}$ & $34.87 \pm 2.63^{\mathrm{de}}$ & $60.03 \pm 0.12^{\mathrm{b}}$ \\
\hline Diclazuril control & $39.48 \pm 1.40^{\mathrm{a}}$ & $53.60 \pm 0.06^{\mathrm{a}}$ & $73.50 \pm 1.44^{\mathrm{a}}$ \\
\hline Infectedamprolium+ethopabate treated & $31.33 \pm 1.41^{\mathrm{b}}$ & $46.17 \pm 2.45^{\mathrm{bc}}$ & $62.83 \pm 1.88^{\mathrm{b}}$ \\
\hline Infected toltrazuril treated group & $25.83 \pm 3.09^{\mathrm{bc}}$ & $39.70 \pm 3.07^{\mathrm{cd}}$ & $61.77 \pm 2.92^{\mathrm{b}}$ \\
\hline
\end{tabular}

Means: Mean of 3 replicates (10 birds / replicate).

$\mathrm{a}_{\mathrm{b}} \mathrm{cde}=$ Means in the same column with different superscripts are significantly different at $\mathrm{P} \leq 0.05$ 
TABLE 5. Weekly FCR post $E$. tsunodai infection.

\begin{tabular}{|l|c|c|c|}
\hline \multirow{2}{*}{ Treatments } & \multicolumn{3}{c|}{ Weekly FCR } \\
\cline { 2 - 4 } & $\mathbf{1}^{\text {st }}$ Week & $2^{\text {nd }}$ Week & $3^{\text {rd }}$ Week $^{*}$ \\
\hline Negative control & $1.51 \pm 0.20^{\mathrm{bc}}$ & $2.42 \pm 0.20^{\mathrm{c}}$ & $2.15 \pm 0.09^{\mathrm{cd}}$ \\
\hline Positive control & $2.51 \pm 0.25^{\mathrm{a}}$ & $3.33 \pm 0.20^{\mathrm{a}}$ & $2.67 \pm 0.12^{\mathrm{a}}$ \\
\hline Infected salinomycine treated & $1.96 \pm 0.28^{\mathrm{ab}}$ & $3.18 \pm 0.10^{\mathrm{ab}}$ & $2.49 \pm 0.15^{\mathrm{ab}}$ \\
\hline Salinomycine control & $1.49 \pm 0.10^{\mathrm{bc}}$ & $2.33 \pm 0.12^{\mathrm{c}}$ & $1.99 \pm 0.03^{\mathrm{d}}$ \\
\hline Infected diclazuril treated & $1.79 \pm 0.17^{\mathrm{bc}}$ & $2.69 \pm 0.24^{\mathrm{c}}$ & $2.45 \pm 0.12^{\mathrm{abc}}$ \\
\hline Diclazuril control & $1.24 \pm 0.22^{\mathrm{c}}$ & $2.24 \pm 0.06^{\mathrm{c}}$ & $1.89 \pm 0.11^{\mathrm{d}}$ \\
\hline $\begin{array}{l}\text { Infected amprolium }+ \text { ethopabate } \\
\text { treated }\end{array}$ & $1.53 \pm 0.12^{\mathrm{bc}}$ & $2.59 \pm 0.24^{\mathrm{c}}$ & $2.34 \pm 0.14^{\mathrm{bc}}$ \\
\hline Infected toltrazuril treated group & $1.74 \pm 0.17^{\mathrm{bc}}$ & $2.64 \pm 0.25^{\mathrm{bc}}$ & $2.44 \pm 0.01^{\mathrm{abc}}$ \\
\hline
\end{tabular}

Means: Mean of 3 replicates (10 birds / replicate).

$\mathrm{a}_{\mathrm{b}} \mathrm{d}=$ Means in the same column with different superscripts are significantly different $\mathrm{Pt} \leq 0.05$.

TABLE 6. Weekly body weights (gm) pre and post $E$. tsunodai infection:

\begin{tabular}{|c|c|c|c|c|}
\hline \multirow[b]{2}{*}{ Treatments } & \multicolumn{4}{|c|}{ Weekly body weights (gm) } \\
\hline & $\begin{array}{c}14^{\text {th }} \text { day of age } \\
\text { (day of } \\
\text { infection) }\end{array}$ & $1^{\text {st }}$ Week & $2^{\text {nd }}$ Week & $3^{\text {rd }}$ Week \\
\hline Negative control & $66.61 \pm 0.05^{\mathrm{a}}$ & $98.68 \pm 2.66^{a}$ & $148.18 \pm 0.61^{\mathrm{ab}}$ & $217.95 \pm 0.78^{\mathrm{ab}}$ \\
\hline Positive control & $57.78 \pm 0.59 \mathrm{~b}$ & $71.95 \pm 2.09 \mathrm{~d}$ & $104.75 \pm 4.03^{\mathrm{f}}$ & $154.52 \pm 3.85^{\mathrm{g}}$ \\
\hline Infected salinomycine treated & $62.18 \pm 1.75^{\mathrm{ab}}$ & $80.64 \pm 1.97 \mathrm{c}$ & $115.31 \pm 3.26 \mathrm{e}$ & $167.71 \pm 1.78^{\mathrm{f}}$ \\
\hline Salinomycine control & $57.41 \pm 0.22^{\mathrm{b}}$ & $90.11 \pm 1.99^{b}$ & $141.31 \pm 1.88 \mathrm{bc}$ & $213.61 \pm 1.99 \mathrm{~b}$ \\
\hline Infected diclazuril treated & $60.84 \pm 0.45^{b}$ & $83.80 \pm 0.99^{\mathrm{c}}$ & $118.67 \pm 2.07 \mathrm{de}$ & $178.70 \pm 2.06^{\mathrm{e}}$ \\
\hline Diclazuril control & $58.10 \pm 0.17 \mathrm{~b}$ & $97.58 \pm 1.23^{\mathrm{a}}$ & $151.18 \pm 1.28^{\mathrm{a}}$ & $224.68 \pm 2.73^{a}$ \\
\hline $\begin{array}{l}\text { Infected } \\
\text { amprolium+ethopabate treated }\end{array}$ & $59.97 \pm 0.69 \mathrm{~b}$ & $91.30 \pm 1.30^{b}$ & $137.47 \pm 3.64^{\mathrm{c}}$ & $200.30 \pm 2.90^{c}$ \\
\hline $\begin{array}{l}\text { Infected toltrazuril treated } \\
\text { group }\end{array}$ & $59.28 \pm 0.28^{b}$ & $85.11 \pm 2.74 \mathrm{bc}$ & $124.81 \pm 4.21 \mathrm{~d}$ & $186.58 \pm 2.17^{\mathrm{d}}$ \\
\hline
\end{tabular}

Means: Mean of 3 replicates (10 birds / replicate)

$\mathrm{a}_{\mathrm{b}} \mathrm{d}$ e $\mathrm{f} \mathrm{g}=$ Means in the same column with different superscripts are significantly different at $\mathrm{P} \leq 0.05$.

TABLE 7. Effect of different anticoccidial drugs on sporulation of $E$. tsunodai oocysts.

\begin{tabular}{|l|c|}
\hline \multicolumn{1}{|c|}{ GROUP } & SPORULATION \% \\
\hline Positive control & $80 \%$ \\
\hline Infected salinomycine treated & $60 \%$ \\
\hline Infected diclazuril treated & $50 \%$ \\
\hline Infected amprolium+ethopabate treated & $50 \%$ \\
\hline Infected toltrazuril treated group & $65 \%$ \\
\hline
\end{tabular}

Egypt. J. Vet. Sci. Vol. 47, No.2 (2016) 
Amprolium and ethopabate showed much better results than toltrazuril in all tested parameters except the mortality rate was the same.

Coccidial water treatments were found to be more effective than prophylactic feed additives in almost all tested parameters. Mortality rate was similar in amprolium plus ethpabate, toltrrazuril and diclazuril treated groups. Toltrazuril had the lowest effect on sporulation of oocysts.

\section{Discussion}

Japanese quails are susceptible to various diseases such as coccidiosis which considered as a serious parasitic disease problem limiting quail industry (Seok et al., 2003).

E. tsunodai (cecal coccidiosis) was found to be more pathogenic than $E$. bateri and E. uzura (intestinal coccidiosis) (Tsutsumi and Tsunoda, 1972).

Results of experimental infection with cecal coccidiosis (E. Tsunodai) revealed that the clinical symptoms and mortalities were lower in quails treated with diclazuril in comparison with salinomycin treated quails and this was in agreement with El-Gaos (2014) in chickens.

With regard to lesion score, our results showed that using either diclazuril or salinomycin reduced lesion score as compared with positive control group. This was consistent with Gerhold et al. (2011) in Bob white quails.

Using either salinomycin or diclazuril in our study decreased the mean oocysts count compared with the positive control group. Such results were in agreement with the findings of Hamad (2011) in chickens. Unlike El-Gaos (2014) who found that diclazuril decreased oocyst shedding more than salinomycin in chickens, our results showed that salinomycin caused decrease in oocyst shedding more than diclazuril. This difference might be attributed to the difference of Eimeria species, management system, environment and drug resistance (Umar et al., 2014).

Diclazuril treated birds had higher weight gain when compared with salinomycin treated ones and this was agreed with Hamad (2011) in chickens.

In our study, FCR and mean body weights were better in diclazuril treated quails in comparison with infected non treated quails and salinomycin treated ones. This was similar to Kheirabadi et al. (2008) in chickens.

In our work diclazuril was found to be highly effective in suppression of sporulation percent than salinomycin. This agreed with Verheyen et al. (1989) who concluded that diclazuril resulting in the formation of abnormal thickened,

Egypt. J. Vet. Sci. Vol. 47, No.2 (2016) 
incomplete oocyst wall and necrosis of the zygote which inhibit oocyst sporulation percent.

We found that quails treated with amprolium in addition to ethobapate showed milder clinical signs than toltrazuril treated ones and this agreed with the findings of El-Gaos (2014) who reported that amprolium 20\% treated chickens showed milder clinical signs compared with toltrazuril treated ones.

Our results revealed that amprolium plus ethobapate treated quails showed no mortality. Similarly, Hameed et al. (2012) found that amprolium lowered motalities compared with sulphadimidine sodium in Japanese quails. Toltrazuril treated group had better survival rates and lower mortalities comparing with positive control group. Similar results also were reported by Alassas (2010) in chickens.

We found that lesion score was significantly severe in positive control group compared with both amprolium and ethopabate treated group or toltrazuril treated group. This was consistent with Saied (2010) in chickens.

Both amprolium and ethopabate and toltrazuril treated quails showed oocyst output lower than that of positive control group. Amprolium and ethopabate treated group shed oocyst lower than toltrazuril treated one and this agreed with El-Gaos (2014) in chickens.

Our study revealed that amprolium plus ethopabate treated quails had weight gain higher than that of toltrazuril $(2.5 \%)$ treated ones. This was in agreement with El-Gaos (2014) in chickens. On the other hand, Saied (2010) found that toltrazuril $2.5 \%$ treated chickens had weight gain higher than that of amprolium $20 \%$ treated ones. Ruff et al. (1987) reported that amprolium was not effective for the control of coccidiosis in bob white quails as evaluated by body weight gains and in Japanese quail he found that amprolium was ineffective for control of coccidiosis. This controversal result might be attributed to the differences in the challenged Eimeria species, host species or drug resistance.

FCR of amprolium in addition to ethopabate treated group were better than that of infected non treated group. Similarly, Hameed et al. (2012) found that amprolium treated Japanese quail group had better FCR values in comparison with that of sulphadimidine sodium treated one. FCR of toltrazuril $(2.5 \%)$ treated group were better than that of positive control group. This was in agreement with results obtained by Nawwar (2007) in chickens.

In our study, body weights of amprolium and ethopabate treated group was higher than that of toltrazuril $(2.5 \%)$ treated one. This agreed with El-Gaos (2014) in chickens.

Our results showed that amprolium and ethopabate lowered sporulation 
percent of E. tsunodai oocysts in comparison with positive control group. This was similar to that found by Shakshouk (1984) in chickens. Toltrazuril (2.5\%) treated group showed lower sporulation percent compared with positive control group and this agreed with Sokol et al. (2014) who found that toltrazuril caused a reduction in the percent of sporulated E. tsunodai oocysts in Japanese quails compared with positive control group.

Comparing the efficacy of curative drinking water anticoccidials (amprolium, ethopabate and toltrazuril) and prophylactic feed additive ones (salinomycin and diclazuril), it was clear from our results that effects of curative drinking water medicaments had the preference in relation to most of previous measured parameters.

\section{References}

Adefolabi, T.K. and Chiejina, S. (1987) The faecal coccidial oocyst output of adult small ruminants in Nigeria. Nigerian Veterinary Journal, 16, 1-6.

Alassas, A.Z. (2010) Efficacy of some anticoccidial agents during coccidiosis in chicken. M.V.Sc., Thesis, Pharmacology, Fac. Vet. Med., Mansoura University.

Berto, B.P., Borba H.R., Lima, V.M., Flausino, W., Teixeira-Filho, W.L. and Lopes, C.W.G. (2013) Eimeria spp. from Japanese quails (Coturnix japonica): new characteristic features and diagnostic tools. Pesqui. Vet. Bras., 33 (12), 1441-1447.

Conway, D.P. and Mckenzie, M.E. (1991) "Poultry Coccidiosis Diagnostic and Testing Procedures", $2^{\text {nd }}$ ed., chapter 2, 17- 36

Duszynski, D.W. and Wilber, P.G. (1997) A guideline for the preparation of species descriptions in the Eimeriidae. Journal of Parasitology, 83 (2), 333-336.

El-Gaos, M.I.A. (2014) Studies on coccidial sensitivity to certain drugs. Ph.D. Thesis, Poultry and Rabbit Dis. Depart., Fac. of Vet. Med., Mansoura University, Egypt.

Gerhold, R.W., Fuller, A.L., LoUis, L., Pan, C. and McDougald, L.R. (2011): The efficacy of anticoccidial products against Eimeria spp. in northern bobwhites. Avian Dis., 55 (1), 59-64.

Hamad, E.M.A. (2011) Evaluation of some anticoccidial drugs and coccidial vaccines in prevention of cecal coccidiosis. M.V.Sc., Thesis, Avian and Rabbit Diseases, Fac. Vet. Med., Zag. Univ.

Hameed, A., Khan, M.S., Rehman, A., Khalid, S., and Umair, M. (2012) Therapeutic study on experimentally induced coccidiosis and its effects on different parameters in quails: a randomized controlled trial. Sci. Int. (Lahore), 24 (4), 461-463.

Henddrix, C.M. and Robinson, E.d. (2006) "Diagnostic Parasitology for Veterinary Technicians", $3^{\text {rd }}$ ed. Mosby, Elsevier, 232-236.

Egypt. J. Vet. Sci. Vol. 47, No.2 (2016) 
Johnson, J. and Reid, W.M. (1970) Anticoccidial drugs lesion scoring techniques in battery and floor-pen experiments with chickens. Exp. Parasitol. 28:30-36.

Kheirabadi, P.Kh., Moghadam, Z.A., Abdi, F., Bahonar, A.R. (2008): The effect of administration of anti-coccidial drugs on oocyst shedding and performance in experimental coccidiosis in broiler chickens. Int. J. Vet. Res., 2 (1), $67-73$.

Nawwar, H.M. (2007) Field evaluation of methods of coccidia infection control in some poultry farms in Dakahlia Governorate. Ph.D., Thesis., Parasitol., Fac. Vet. Med., Kafr El-Sheikh Univ.

Norton, C.C. and Joyner, L.P. (1968) Preservation of coccidian. Res. Vet. Sci., 9, 598 600 .

Ruff, M.D., Wilkins, G.C. and Chute, M.B. (1987) Prevention of coccidiosis in bobwhites by medication. Poultry Science, 66 (9), 1437-1445.

Saied, M.H. (2010) Studies on evaluation of recent anticoccidial drugs for prevention and control of chickens coccidiosis. M.V.Sc., Thesis, Poultry Depart., Fac. Vet. Med. Cairo University.

Seok, S., Park, J., Cho, S., Baek, M., Lee, H. and Kim, D. (2003) Coccidian (Eimeria spp.) in small intestine of Japanese quail (Coturnix coturnix japonica). Kor. J. Lab. Ani. Sci., 19 (2), 90-91.

Shakshouk, A.R. (1984) Studies on chicken coccidiosis with special references to drug screening. Master Thesis, Fac. Vet. Med., Alexandria Univ.

Snedecor, G.W. and Cochran, W.G. (1981) "Statistical Methods", $7^{\text {th }}$ ed. Iowa State University, Ames, Iowa, USA.

Sokól, R., Gesek, M., Raś-Noryńska, M. and Michalczyk, M. (2014) Toltrazuril $\left(\right.$ Baycox $\left.{ }^{\circledR}\right)$ treatment against coccidiosis caused by Eimeria sp. in Japanese quails (Coturnix coturnix japonica). Polish Journal of Veterinary Sciences, 17 (3), 465-468.

Soulsby, E.J.I. (1968) Helminthes, Arthropods and protozoa, domestic animals. $7^{\text {th }}$ ed. Bailliere-Tindall, London.

Teixeira, M. and Lopes C.W.G. (2002) Species of the genus Eimeria (Apicomplexa: Eimeriidae) from Japanese quails (Coturnix japonica) in Brazil and E. fluminensis for the preoccupied E. minima of this quail. Revista Brasileira de Ciências Veterinárias, 9, 53-56.

Tsutsumi, Y. (1972) Eimeria tsunodai n.sp. (Protozoa: Eimeriidae). A cecael coccidium of Japanese quails (Coturnix japonica). Jap. J. Vet. Sci., 34,1-9.

Tsutsumi, Y. and Tsunoda, K. (1972) Pathogenicity of Eimeria tsunodai from Japanese quails (Cotumix cotumix japonica) and susceptibility of the coccidium to some drugs. Jap. J. Vet. Sci., 34, 115-121.

Egypt. J. Vet. Sci. Vol. 47, No.2 (2016) 
Umar, H.A., Lawal, I.A., Okubanjo, O.O. and Wakawa, A.M. (2014) Morphometric identification, Gross and Histopathological Lesions of Eimeria species in Japanese quails (Coturnix coturnix japonica) in Zaria, Nigeria. Journal of Veterinary Medicine, Vol. 2014, Article ID 451945, 6 pages. doi:10.1155/2014/451945.

Urquhart, G.M., Armour, J., Duncan, J.L., Dunn, A.M. and Jennings F.W. (2003) "Veterinary Parasitology", $2^{\text {nd }}$ ed. Black Well Science Ltd. Oxford. 276p.

Verheyen, A., Maes, E., Coussement, W., Vanparijs, O., Lauwers, F., Vlaminckx, E and Marsboom, R. (1989) Ultrastructural evaluation of the effects of diclazuril on the endogenous stages of Eimeria maxima and Eimeria brunetti in experimentally inoculated chickens. Parasitol. Res., 75, $604-610$.

كفاعة بعض مضادات الكوكسيديا ضد الكوكسيديا الاعوريه (ايميريا تسونوداي) المجرعه معمليا في طائر السمان الياباني

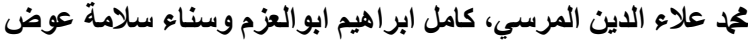

$$
\begin{aligned}
& \text { قسم امر اض الطيور والارانب - كلية الطب البيطري - جامعة المنصورة - }
\end{aligned}
$$

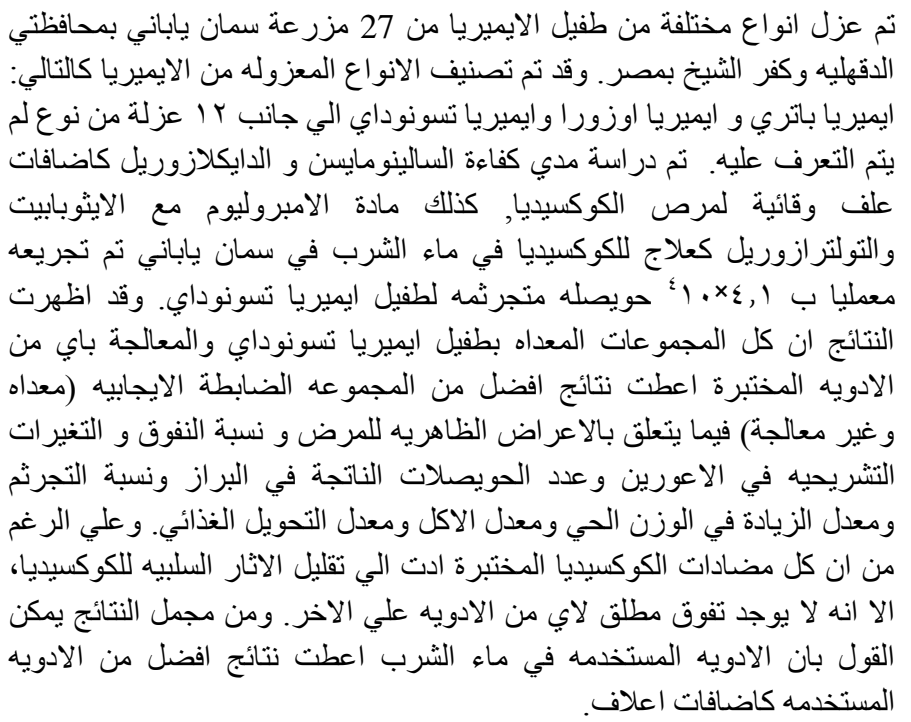

